\title{
Medullo-Epithelioma of the Ciliary Body
}

\begin{tabular}{|l|l|l|}
\hline A. & Hamburg \\
\hline
\end{tabular}

Hilversum

In a man of 50 the right ej^e was removed on account of a diagnosis of melanoblastoma of the ciliary body. Histological investigation revealed that the tumour originated from the pig-mented epithelium of the ciliary body and possibly from the iris. There was a slight infiltrative growth in the area.

From the literature it appeared that opinions about the nature of the-extremely rare-epithelial tumours that occur among adults and the manner in which they originate are divided. There is more agreement about the embryonic form (diktyoma). It is true

476 Hamburg, Medullo-Epithelioma of the Ciliary Body

that the embryonic and adult forms are grouped under the term medullo-epithelioma. The histological picture of the adult form, also called malignant epithelioma, is so varied that no typical description of it can be given. Moreover, a number of these tumours have been described as issuing from senile, inflammatory or pseudo-adenomatous epilhelial hyperplasias while others are said to have developed spontaneously.

About one thing there is agreement, i.e. no metastases occur, while the local infiltrative growth is slight. Clinically the spontaneous tumours reveal themselves in a gradual decrease of visual acuity. During examination a tumour is found in the neighbourhood of the anterior chamber angle, together with a more or less marked clouding of the lens. This is not particularly important since the therapeutic consequences are the same in both cases, melanoblastoma and medulloepithelioma.

Discussion

Dr. Manschot: According to the speaker medullo-epithelioma originates from the pigmented epithelium of the ciliary body. The strongly pigmented tumour tissue shown on the slides would indicate this. In the literature it is generally agreed that both the embryonic form (diktyoma) and the adult form, as demonstrated here, find their origin in the unpigmented inmost layer of the epithelium of the ciliary body, thus in the inner wall of the eye cup. Wolter 1 , with his silver carbonate dye, found an excess of primitive nerve fibers in a similar tumour of the adult type. This is, however, only to be explained by its origin in the inmost layer of the eye cup. Hamburg: I have not read the article referred to by Dr. Manschot but Reese 2 also suggests that all epithelial tumours of the ciliary body, the pigmented ones included, originate from the inner epithelial layer. I wish to express my thanks for this information.

1 Wolter

J. R.: Amer. J. Ophthal. 46: 19 (1958).

2 Reese

A. B.: Tumours of the Eye (Cassel

London 1951). 